\title{
Cardiorenal Syndrome Type 5: Clinical Presentation, Pathophysiology and Management Strategies from the Eleventh Consensus Conference of the Acute Dialysis Quality Initiative (ADQI)
}

\author{
Ravindra L. Mehta ${ }^{a} \cdot$ Hamid Rabb ${ }^{b}$ Andrew D. Shaw ${ }^{c}$. \\ Kai Singbartld Claudio Ronco $^{e} \cdot$ Peter A. McCullough ${ }^{f}$. \\ John A. Kellum ${ }^{9} \cdot$ for the Acute Dialysis Quality Initiative (ADQI) \\ 11 Working Group
}

${ }^{\text {a }}$ Department of Medicine, University of California San Diego, San Diego, Calif., USA; ${ }^{b}$ Department of Medicine, Johns Hopkins University, Baltimore, Md., USA; ' Department of Anesthesiology, Duke University Medical Center, Durham, N.C., USA; ${ }^{d}$ Department of Anesthesiology, Penn State College of Medicine, Milton S. Hershey Medical Center, Hershey, Pa., USA; ${ }^{\mathrm{e}}$ Department of Nephrology, International Renal Research Institute (IRRIV), San Bortolo Hospital, Vicenza, Italy; ${ }^{f}$ St. John Providence Health System, Warren, Mich., Providence Hospitals and Medical Centers, Southfield and Novi, Mich., St. John Macomb Oakland Center, Madison Heights, Mich., St. John Hospital and Medical Center, Detroit, Mich., USA; ${ }^{9}$ Clinical Research, Investigation, and Systems Modeling of Acute IIIness (CRISMA) Center, Department of Critical Care Medicine, University of Pittsburgh, Pittsburgh, Pa., USA

\begin{abstract}
Cardiorenal syndrome type 5 (CRS-5) includes conditions where there is a simultaneous involvement of the heart and kidney from a systemic disorder. Given the wide spectrum of diseases that contribute to CRS-5, several pathophysiological mechanisms are invoked representing the response of the heart and kidney to the contributing disorder that is ongoing. The nature, duration and the underlying condition of the heart and kidney strongly influence the clinical course of CRS-5. In this paper we discuss the pathophysiology of CRS-5 in the setting of sepsis as a model system for CRS-5 providing a brief overview of strategies for monitoring and therapeutic intervention. We offer a framework for reference for considering other disorders leading to CRS-5 where the development of cardiac and renal dysfunction is more insidious.

Copyright $\odot 2013$ S. Karger AG, Basel
\end{abstract}


Type 5 cardiorenal syndrome (CRS-5) occurs when an overwhelming insult leads to the simultaneous development of acute kidney injury (AKI) and acute cardiac dysfunction. In the literature, CRS- 5 most commonly encompasses a wide spectrum of disorders that acutely involve the heart and kidney such as sepsis and drug toxicity where both the heart and the kidney are involved secondarily to the underlying process [1]. CRS-5 may develop in the setting of previously impaired organ function or when there is no discernible evidence of prior abnormality. The sequence of organ involvement can vary depending on the acuity and nature of the underlying disorder. The time sequence for developing CRS- 5 depends on the underlying disease and is influenced by the underlying level of cardiac and renal function.

Given the multitude of contributing factors and the time sequence of events in CRS-5, it is challenging to identify the underlying pathophysiological mechanisms and develop a strategy for diagnostic and therapeutic intervention. In this paper we provide a framework for considering the pathophysiology of CRS-5 using severe sepsis and septic shock as model disorders in which we discuss the relevant systems pathophysiology, molecular pathways involved diagnostic approach and therapeutic considerations.

\section{Methods}

We performed a systemic review of the literature pre-conference, as described elsewhere. Specifically, we used the terms 'cardiorenal syndrome type 5' and 'acute kidney injury', combined with 'sepsis', 'liver failure', 'lupus', 'pathophysiology' and 'mechanisms'. In view of the volume of the retrieved literature, only representative publications are cited in this review. Furthermore, we opted to focus this summary to the clinical scenario of acute CRS-5 using sepsis as a model system.

\section{Results}

Based on the literature identified prior to the conference, the following key questions were considered:

1. What is the underlying pathophysiology of CRS-5?

a. What are the predisposing and modifying factors?

b. What are the direct effects of the underlying disorder on the heart and kidney?

c. Does involvement of one organ contribute to dysfunction in the other?

d. Which cellular and molecular mechanisms are involved? 
e. What is the influence of process of care on pathophysiology, e.g. fluid resuscitation?

2. What are the best techniques for the diagnosis and monitoring of CRS-5?

a. To evaluate the nature and severity of cardiac and renal damage.

b. To assess progression and resolution of organ dysfunction.

3. What are the targets and best strategies for therapeutic intervention?

a. Management of underlying disease.

b. Treatment of cardiac and renal dysfunction.

\section{Q1. What Is the Underlying Pathophysiology of Cardiorenal Syndrome Type 5?}

\section{a. What Are the Predisposing and Modifying Factors?}

The pathophysiology of CRS-5 depends on the underlying disease state, the time frame for development and the context in which it is encountered. Acute CRS-5 results from systemic processes, e.g. sepsis, infections, drugs, toxins and connective tissue disorders such as lupus, Wegener's granulomatosis, and sarcoidosis. While there is some overlap in these conditions, to a large extent, the time frame for development and resolution represent the underlying pathophysiological mechanisms. For instance, in sepsis-induced acute CRS-5 there is a fulminant disease process with dramatic impact on both kidney and heart, with obvious clinical manifestations. In contrast, in cirrhotic liver disease, CRS-5 has a more insidious onset and the kidney and cardiac dysfunction may develop slowly until a 'tipping point' is reached and full-blown decompensation occurs. Several factors influence the course of CRS-5 (table 1). The inducing events and the immunological and physiological responses are conditioned by the underlying condition of the heart and kidney. Although the mechanisms invoked in acute and chronic CRS-5 are somewhat different (fig. 1,2), the nature, severity and duration of organ dysfunction are also influenced by the process of care to manage the condition (table 1 ).

In order to understand the underlying pathophysiology and identify opportunities for intervention it is helpful to consider the temporal sequence of CRS5 as characterized by the natural history and management of the underlying disorder (table 2). Acute CRS-5 develops along a temporal framework consisting of hyperacute ( $0-72 \mathrm{~h}$ after diagnosis), acute (3-7 days), subacute (7-30 days) and chronic (30+ days) phases (fig. 3). Most published human literature refers to the hyperacute phase, since this has been the focus of most of the interventional clinical trials conducted to date in sepsis. Chronic CRS-5, e.g. in cirrhotic patients, similarly demonstrates distinct phases of pre-ascites, diuret- 
Table 1. Predisposing and modifying factors influencing development of sepsis induced CRS-5

\begin{tabular}{|c|c|}
\hline Patient attributes & Process of care \\
\hline Inducing event and immunological response & $\begin{array}{l}\text { Resuscitation } \\
\text { - Volume effect on tissue edema and } \\
\text { renal venous hypertension } \\
\text { - } \text { Abdominal compartment syndrome } \\
\text { - } \text { Glycocalyx }\end{array}$ \\
\hline Underlying condition of heart and kidney & $\begin{array}{l}\text { Search for primary focus } \\
\text { - Contrast use }\end{array}$ \\
\hline \multirow[t]{3}{*}{$\begin{array}{l}\text { Physiological responses } \\
\text { - Peripheral vasodilation } \\
\text { - } \quad \text { Compensatory hormonal response } \\
-\quad \text { Increased vascular permeability } \\
-\quad \text { Mitochondrial dysfunction and tissue hypoxia } \\
-\quad \text { Cardiac filling } \\
-\quad \text { Renal hypoperfusion }\end{array}$} & $\begin{array}{l}\text { Drug dosing } \\
\text { - Antibiotics } \\
\text { - Sedatives } \\
\text { - Vasopressors and ionotropes }\end{array}$ \\
\hline & $\begin{array}{l}\text { General supportive care } \\
\text { - Fluids } \\
\text { - Inotropes, pressors }\end{array}$ \\
\hline & $\begin{array}{l}\text { Specific interventions } \\
- \text { Ventilator } \\
\text { - Surgery } \\
- \text { RRT }\end{array}$ \\
\hline
\end{tabular}

ic responsive and diuretic refractory ascites prior to the development of hepatorenal syndrome although the time sequence is quite variable (fig. 4). In most cases of chronic CRS-5 there is usually a precipitating event that brings the condition to attention. For instance in cirrhosis this is often an infection such as spontaneous bacterial peritonitis. Thus an acute CRS-5 can be superimposed on a chronic indolent process. Although we recognize the importance of all phases of CRS-5, we focus here on the pathophysiology of acute CRS-5 because of its immediate relevance for intensivists, nephrologists and cardiologists.

\section{b. What Are the Direct Effects of Underlying Disorder on the Heart and}

\section{Kidney?}

With respect to the cardiorenal syndrome, changes in systems physiology during sepsis can result from the systemic effects of sepsis itself, from the septic injury to 'systemic pathways', or from the organ cross-talk between injured heart and kidney. 


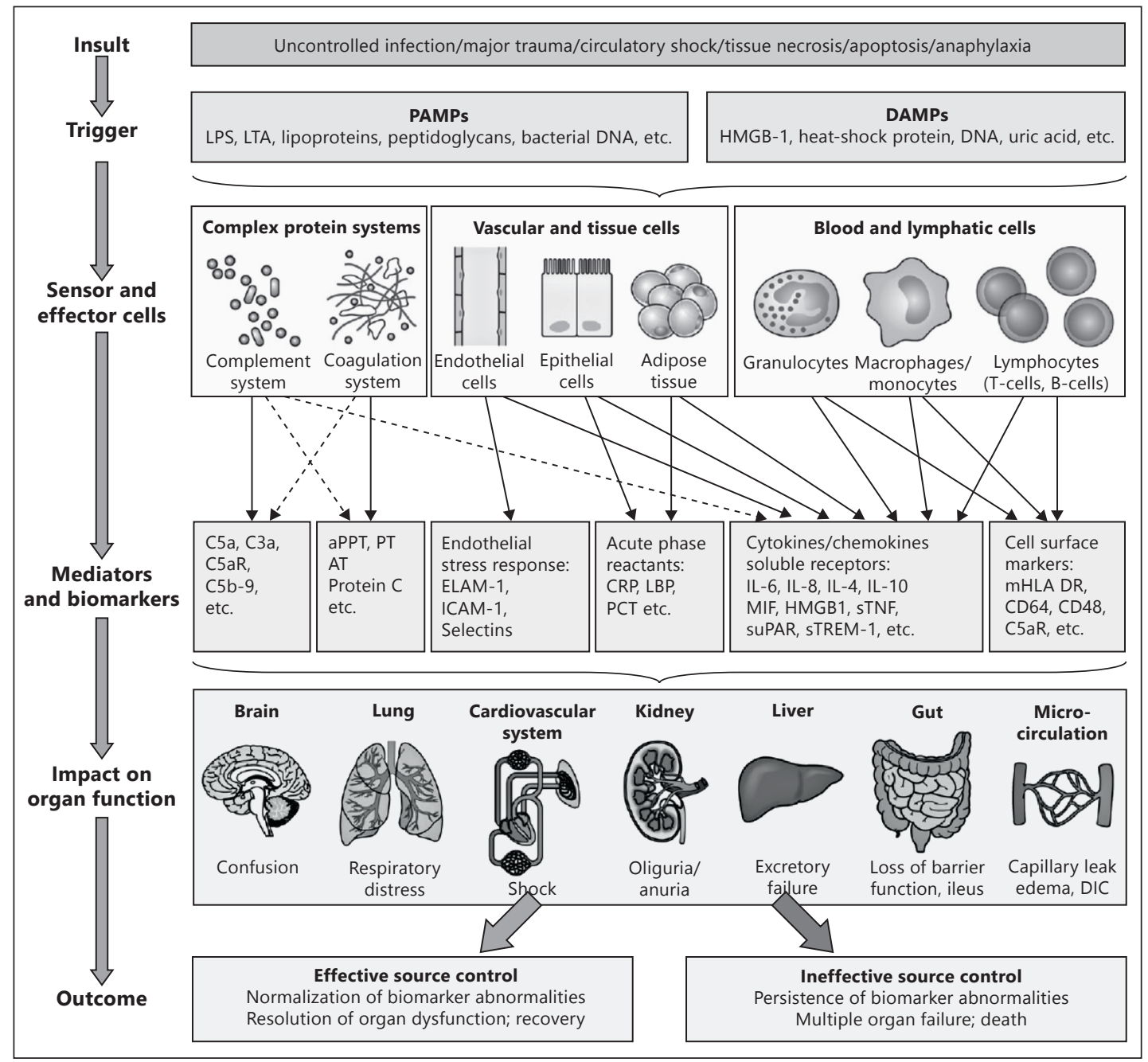

Fig. 1. Pathophysiology of sepsis-induced organ dysfunction. From [49] with permission.

An overall key feature of sepsis is the dissociation between the systemic circulation and the microcirculation in various organs [2]. Especially in the early phases of sepsis, profound microcirculatory changes can develop, despite apparently normal systemic hemodynamics [1-3]. Microcirculatory changes, such as lower blood flow velocities and heterogeneous perfusion patterns, strongly correlate with morbidity and mortality rates [4].

Septic Injury of the Heart (Septic Cardiomyopathy). Septic cardiomyopathy (SCM) represents one of the major predictors of morbidity and mortality of sepsis and is present in nearly $50 \%$ of all patients $[5,6]$. Sepsis can cause both the 


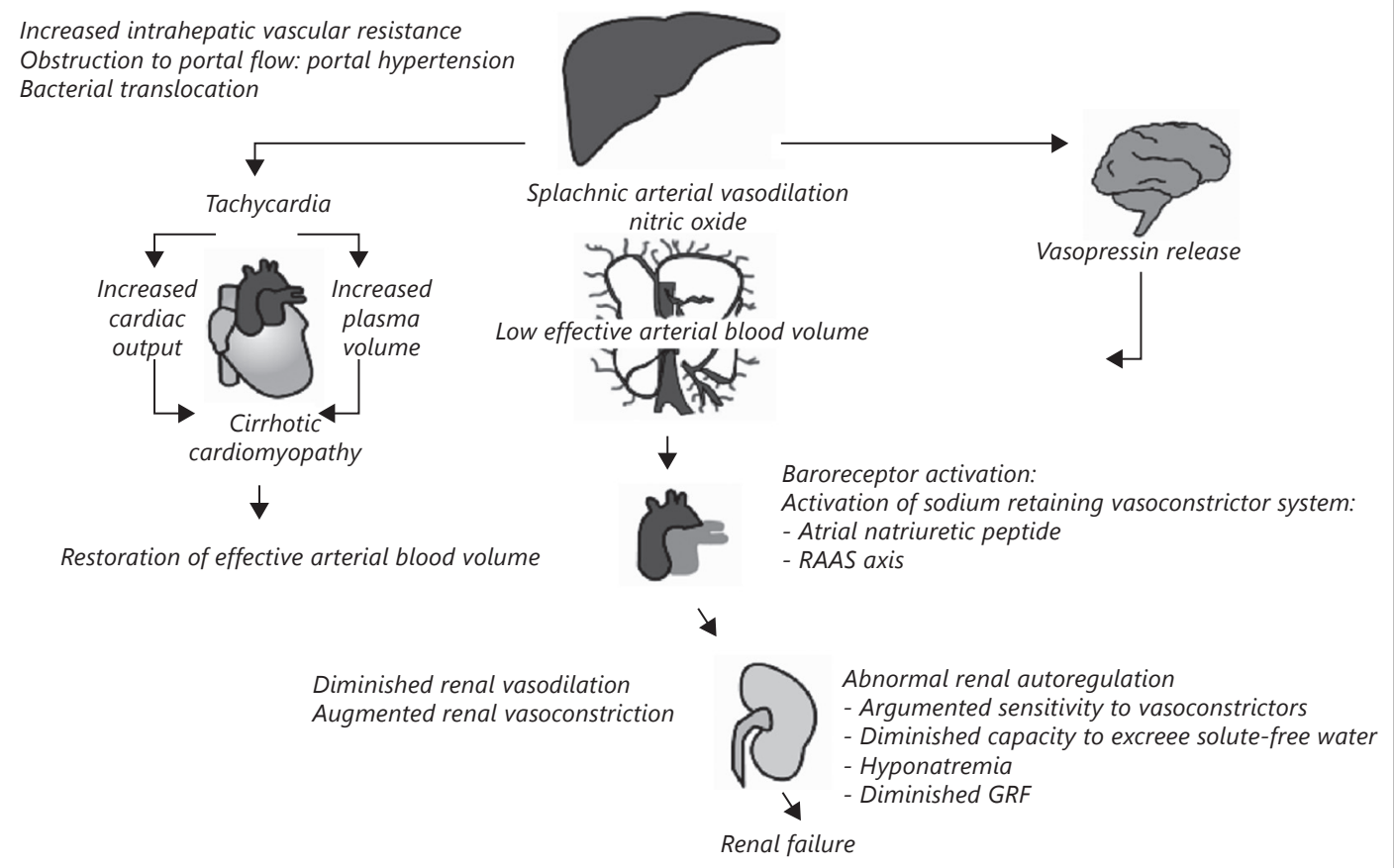

Fig. 2. Pathophysiology of cirrhosis-induced CRS-5 [from 91, with permission].

Table 2. Temporal considerations in pathophysiology of CRS-5

\begin{tabular}{|c|c|c|}
\hline Attribute & CRS-5 acute (sepsis) (fig. 1) & CRS-5 chronic (cirrhosis) (fig. 2) \\
\hline Time for organ dysfunction & Short: hours to days & Long: weeks to months \\
\hline Underlying organ function & $\begin{array}{l}\text { May be superimposed on under- } \\
\text { lying cardiac and kidney disease }\end{array}$ & $\begin{array}{l}\text { Heart and kidney have adaptive responses that fail over } \\
\text { time }\end{array}$ \\
\hline $\begin{array}{l}\text { Sequence of organ } \\
\text { involvement }\end{array}$ & $\begin{array}{l}\text { Generally simultaneous or in } \\
\text { close proximity to each other }\end{array}$ & $\begin{array}{l}\text { One organ precedes the other, e.g. cardiac dysfunction } \\
\text { precedes renal in cirrhosis }\end{array}$ \\
\hline Underlying disease & Systemic event contributes to CRS5 & $\begin{array}{l}\text { Precipitating events can transition to an acute deteriora- } \\
\text { tion in CRS5, e.g. Gl bleeding can precipitate hepatorenal } \\
\text { syndrome }\end{array}$ \\
\hline Pathophysiology & Direct effects on organs & Failure of adaptive responses over time \\
\hline Mechanisms & Determined by underlying disease & Determined by adaptive changes \\
\hline Reversibility & $\begin{array}{l}\text { Possible with control of sepsis and } \\
\text { organ support }\end{array}$ & $\begin{array}{l}\text { Limited unless there is replacement of diseased organ, } \\
\text { e.g. liver transplant }\end{array}$ \\
\hline
\end{tabular}




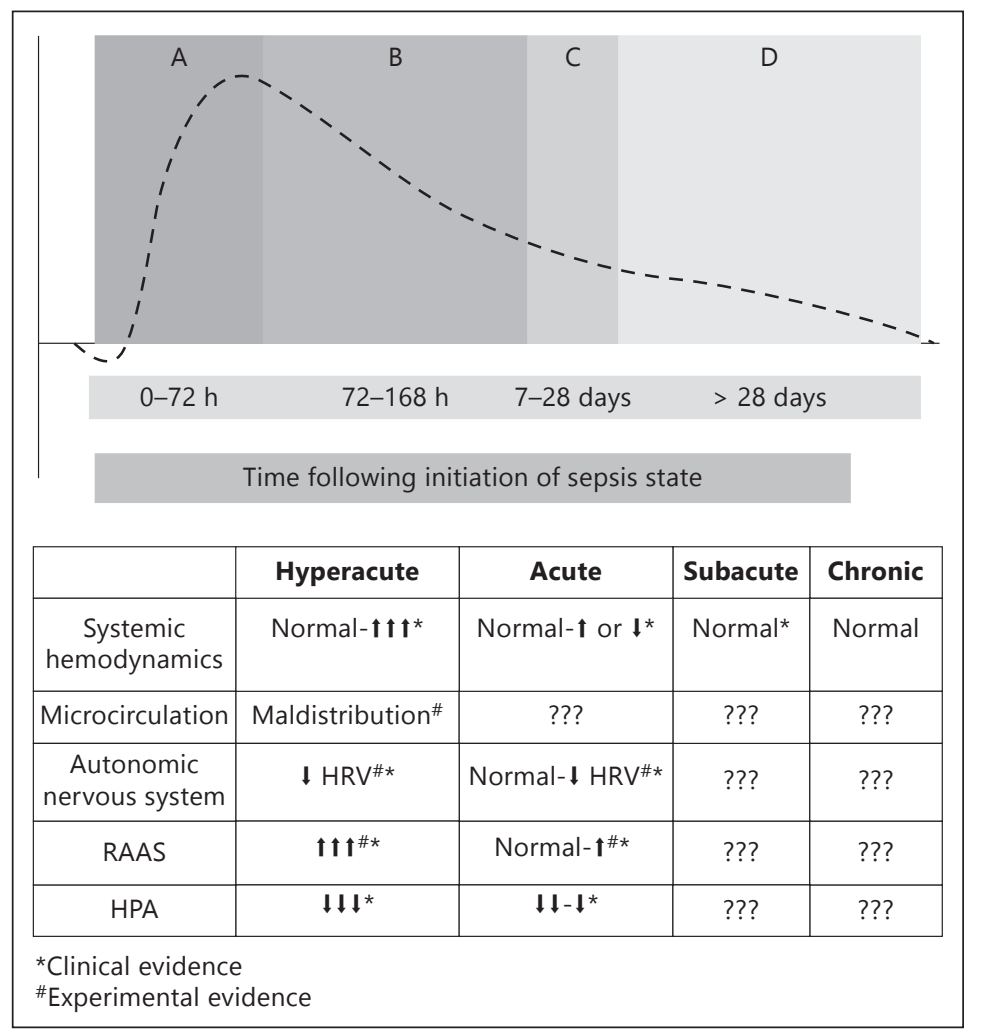

Fig. 3. Pathophysiologic changes in sepsis-induced acute CRS-5. Organ dysfunction in sepsis can be considered in several different phases that reflect the severity of the disease. The underlying pathophysiological mechanisms are different in each phase and thus provide specific opportunities for targeted diagnostic and therapeutic strategies. Phase A: 0-72 $\mathrm{h}=$ hyperacute phase; phase $\mathrm{B}: 72-168 \mathrm{~h}=$ acute phase; phase $\mathrm{C}: 7-28$ days = subacute phase; phase $\mathrm{D}:>28$ days $=$ chronic. RAAS = Renin-angiotensin-aldosterone system; $\mathrm{HPA}=$ Hypothalmus-pituitary gland-adrenal gland axis; HRV = Heart rate variation.

left and the right ventricle to dilate and develop decreased ejection fractions, leading to impaired responsiveness to fluid and catecholamine treatment [7]. Although SCM can become so severe mimicking cardiogenic shock, it is reversible within 7-10 days in most cases [5, 6]. Moreover, as long as hypovolemia is corrected, tachycardia and reduced vascular tone allow for a maintained or even increased cardiac output in many patients. Contrary to early wisdom, myocardial blood flow or energy metabolism do not seem to play a role in SCM [8]. On the other side, myocardial depressants, including pro-inflammatory cytokines and complement factors, have emerged as crucial factors in the development of SCM [9-11]. 


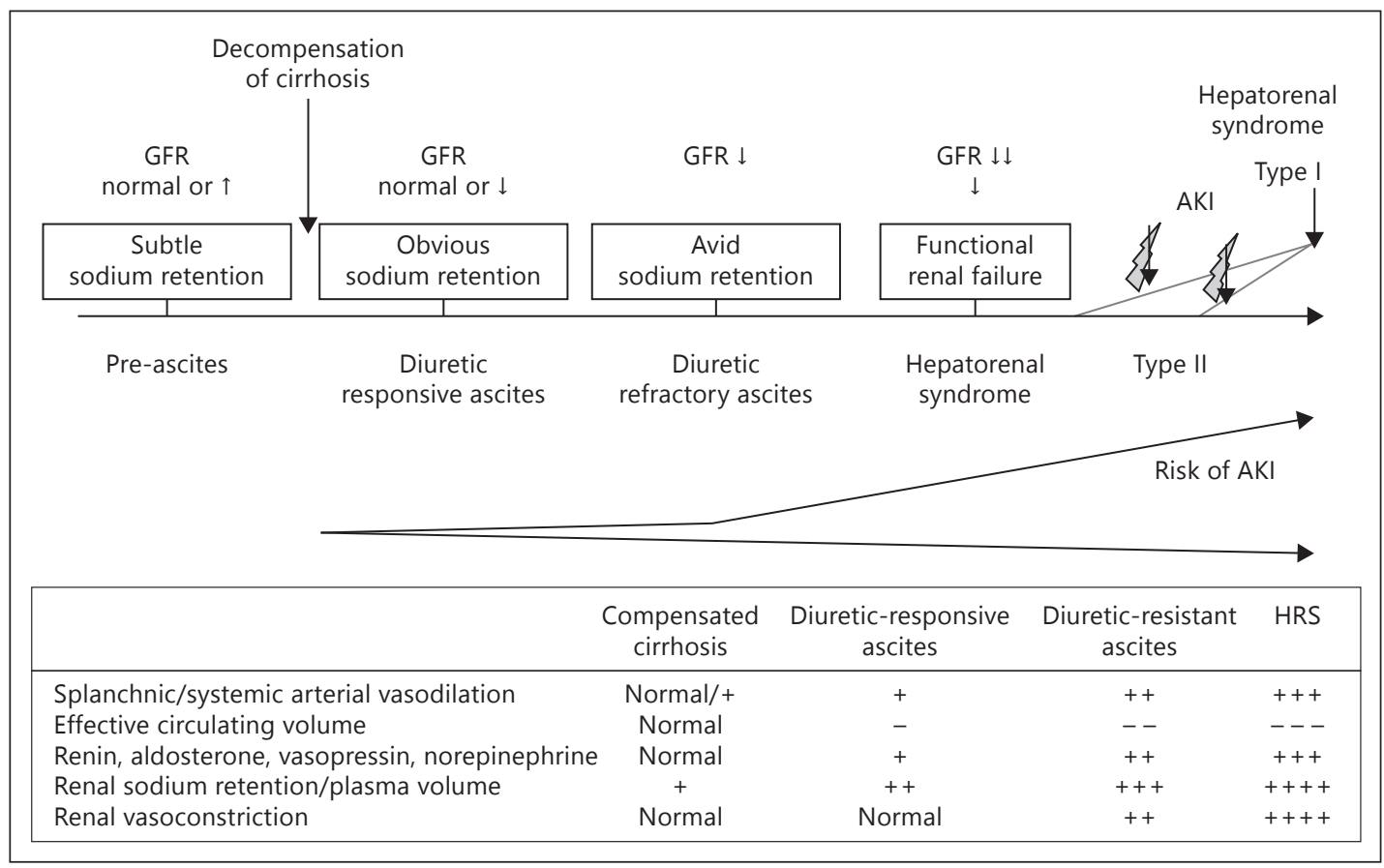

Fig. 4. Chronic CRS-5 hemodynamic changes during different stages of cirrhosis. From [92] with permission.

Septic Kidney Injury. In addition to cellular and molecular changes in the kidney, which are discussed below, there are also alterations to the intra-renal blood flow during sepsis. These changes are uncoupled from the systemic hemodynamic changes occurring during sepsis [12-14]. The exact nature of these changes, however, remains highly controversial. A lack of sound clinical data as well as the heterogeneous quality and limited applicability of various animal models do not allow us to develop a clear concept. A recent experimental study comparing two different sepsis models in pigs might be the first step to overcome this problem [12]. Both experimental design and findings resemble the heterogeneity seen in septic patients very well. Irrespective of systemic hemodynamics (normo- or hyperdynamic), only animals that developed septic AKI also demonstrated an increase in renal vascular resistance. Moreover, only animals with septic AKI also exhibited early rises in pro-inflammatory cytokines, e.g. IL-6, and in markers of oxidative stress. The excellent agreement of these findings with those from observational clinical studies $[15,16]$ allows us to formulate strong hypotheses that require further testing in larger clinical studies. 
Neurogenic and Endocrine Alterations. Sepsis also affects central structures or pathways, including the autonomic nervous system (ANS), the renin-angiotensin-aldosterone system (RAAS) and the hypothalamus-pituitary gland-adrenal gland axis (HPA), which in turn impact cardiac and/or renal function. Sepsis causes ANS dysfunction [17-19], the severity of which correlates with morbidity and mortality. A hallmark of autonomic dysfunction during sepsis is the decreased heart rate variability. A decrease or even loss of heart rate variability (HRV) during sepsis is associated with the release of inflammatory mediators, e.g. IL-6, IL-10 and CRP. Data with respect to kidney-related changes in ANS during sepsis is limited to animal studies. Here, sepsis-induced changes in renal sympathetic nerve activity did not seem to affect renal blood flow [20].

Sepsis activates the RAAS, reflecting the body's attempt to restore and maintain a sufficient blood pressure. Although counterintuitive, recent experimental and limited clinical data suggest that blockade of the RAAS might be beneficial, as RAAS activation has also been implicated in endothelial dysfunction, organ failure and even mortality during severe sepsis [21-24]. Experimental studies further suggest deleterious effects of RAAS activation on renal function during sepsis [13, 25-27]. The administration of ACE inhibitors improves creatinine clearance and urine output during experimental bacteremia; the application of selective angiotensin II type 1 receptor antagonist improves renal blood flow and oxygenation during experimental endotoxemia.

Sepsis causes complex alterations of HPA and glucocorticoid signaling, leading to severe adrenal insufficiency in some patients [28]. Adrenal insufficiency in turn gives rise to increased production of pro-inflammatory cytokines, free radicals and prostaglandins as well as inhibition of chemotaxis and expression of adhesion molecules. Administration of moderate-dose glucocorticoids for 7 days can reduce the need for vasopressors and intensive care unit (ICU) length of stay $[29,30]$.

There is no sound data regarding the effects of adrenal insufficiency on renal function.

\section{c. Does Involvement of One Organ Contribute to Dysfunction in the Other?} Among the most provocative yet complex questions in multiorgan failure is to determine the precise role of the failing heart or kidney in dysfunction of either organ. The plethora of effects of sepsis on the function of various organs, including heart and kidneys, makes it very difficult to differentiate between the effects of sepsis itself and the effects of inter-organ cross-talk. However, some effects of inter-organ cross-talk can be postulated from the sepsis-specific pathophysiology: (i) a reduced cardiac output will lead to reduced renal perfu- 
sion, further aggravating sepsis-induced kidney injury; (ii) fluid overload due to AKI can lead to CHF in already dilated and hypocontractile heart, and (iii) AKIinduced metabolic acidosis can impair contractility and increases the heart rate, worsening myocardial workload.

Limited experimental data allow us to hypothesize that AKI exerts cardiodepressing effects via remote induction of pro-inflammatory and pro-apoptotic pathways [31].

Beyond the hemodynamic effects of the failing heart on renal circulation, there are cardiac changes due to impaired fluid clearance by the kidney. However, AKI itself is well established experimentally to lead to distant organ function [32]. In a murine model, AKI led to decreased cardiac contractility and apoptosis, which was partially abrogated by anti-TNF treatment [31]. AKI also led to cardiac hypertrophy [33]. AKI can cause an increase in cardiac macrophages [34]. Better studied has been the effect of AKI on lung function, where there is an increase in microvascular permeability, endothelial cell dysfunction, and caspase-mediated apoptosis [35]. A well-orchestrated molecular response occurs in lung independent of volume overload [36], and this signature has been localized to endothelial cell [37]. The brain is particularly affected by AKI, and this may in turn affect systemic neuroendocrine response in sepsis [38]. Though this is a particularly challenging area of research, it is potentially among the most important as this could decrease the severe mortality associated with combined acute heart and kidney failure by guiding extracorporeal support devices which cleanse circulating factors that enable deleterious interorgan cross-talk.

\section{d. Which Cellular and Molecular Mechanisms Are Involved?}

During combined acute cardiac and kidney dysfunction, such as in sepsis, there are marked cellular and molecular changes in each organ. There is an important time-specific pattern for these changes, but this aspect has not been sufficiently studied. Furthermore, it is difficult, except using highly controlled experimental models, to tease out the role of the primary infection versus the role of each failing organ on the other.

Activation and induction of cytokines, leukocytes, toll receptors is well established in heart and kidney during sepsis [39]. Notable are pro-inflammatory cytokines TNF and IL-6, while IL-10 has been implicated as anti-inflammatory. Macrophages, neutrophils, lymphocytes, and more recently $\mathrm{T}$ regs, have been implicated. There is an increasing body of evidence supporting the role of danger and pattern-associated receptors, DAMPS and PAMPs, and Toll receptors are among the most important pathophysiologically (fig. 1) [40]. Abnormalities in oxidative stress are likely key mechanisms as well. These range 
from mitochondrial dysfunction [41]), to alteration in antioxidant stress enzymes, particularly those mediated by the Nrf2-Keap pathway [42].

Given the fundamental contractile function of the heart, there are specific molecular pathways that are deranged. Cardiac muscle protein expression, particularly actin and myosin are abnormal in sepsis. Membrane-associated proteins, especially dystrophin, regulate cell shape, mechanical strength and cardiomyocyte transduction of contractile force. The mean amounts of dystrophin and associated glycoproteins in significant reduced in septic hearts [39]. Intercalated discs are the sites where cardiomyocytes connect to each other and both ensure mechanical coupling plus enable electrical signal propagation. Components of intercalated discs including connexin- 43 and $\mathrm{N}$-cadherin are markedly reduced in sepsis, with dehiscence of the junctions during sepsis. L-type calcium currents, important in cardiac function, are reduced during septic shock [43].

In the kidney, geared towards filtration, fluid and electrolyte regulation, there are specific tubular changes after sepsis. Lipopolysaccharide directly alters $\mathrm{HCO}_{3}{ }^{-}$transport through toll receptors, leads to abnormalities in acidification of urine [44]. Sepsis leads to a decline in autophagy in the proximal tubule, which in turn contributes to proximal tubule function [45]. LPS also modifies the important glomerular protein megalin, which can contribute to an increase in urine albumin [46].

e. What Is the Influence of Process of Care on Pathophysiology?

- The management of sepsis can contribute to the development of CRS-5. Fluid resuscitation coupled with the increased vascular permeability can lead to organ and tissue edema and result in an abdominal compartment syndrome with an increase in renal venous pressure and reduced renal perfusion [47]. The utilization of contrast agents for imaging studies can similarly lead to myocardial depression and tubular toxicity. There is a prolonged half life of drugs due to a reduction in hepatic and renal clearance that can result in organ toxicity. All these factors can influence the development and course of CRS-5.

\section{Q2. What Are the Best Techniques for the Diagnosis and Monitoring of CRS-5?}

In considering a diagnostic approach to CRS-5 it is important to decide what the clinician is trying to achieve at each stage of the disease process. Initially the emphasis is on diagnosis (of severe sepsis and septic shock), organ function 
assessment, risk prediction and treatment guidance. Attention then turns to monitoring organ function (using both physiological and biochemical tools) while the patient is stabilized and further organ dysfunction prevented where possible. Assuming the patient begins to recover, the emphasis turns to assessment of residual organ function impairment and assessment of the long-term impact of the disease on the patient's life. Here we discuss these in the context of CRS-5.

\section{a. To Evaluate the Nature and Severity of Cardiac and Renal Damage}

Diagnosis of Severe Sepsis and Septic Shock. For practical purposes, when systemic inflammation occurs in the setting of known or suspected infection, the diagnosis of sepsis is made. Once organ dysfunction develops the diagnosis progresses to severe sepsis, and when the systemic blood pressure cannot be maintained without vasoactive therapy then septic shock is present. Systemic inflammation is usually said to be present when two or more of the systemic inflammatory response syndrome (SIRS) criteria are present: body temperature $<36^{\circ} \mathrm{C}\left(96.8^{\circ} \mathrm{F}\right)$ or $>38^{\circ} \mathrm{C}\left(100.4^{\circ} \mathrm{F}\right)$; heart rate $>90$ beats $/ \mathrm{min}$; tachypnea ( $>20$ breaths/min) or arterial partial pressure of carbon dioxide $<32 \mathrm{~mm} \mathrm{Hg}$; white blood cell count $<4 \times 10^{9}$ cells $/ 1$ or $>12 \times 10^{9}$ cells $/ 1$; or the presence of $>10 \%$ immature neutrophils (band forms) although many sepsis trials have required three of these criteria to be present in order to increase the specificity of the diagnosis [48]. Reinhart et al. [49] present an excellent review of the current state of the art regarding biomarkers for the diagnosis and management of sepsis - readers are referred to this for detailed information regarding the place of candidate markers such as lipopolysaccharide binding protein, procalcitonin, acute phase reactants, cytokines, chemokines and '-omic' techniques including the use of microarrays for pathogen determination and treatment.

Assessment of Cardiac and Renal Function. Assessment and monitoring of cardiac dysfunction in CRS-5 relies on the same techniques as in other causes of acute myocardial dysfunction. Biochemical assays based on natriuretic peptides and troponins provide evidence of cardiac chamber morphological change and myocyte leak respectively. Traditional markers such as leukocytosis and C-reactive protein are non-specific for myocardial assessment, and most clinicians rely on functional monitoring to guide assessment and management. The cardiomyopathy of sepsis is complex and incompletely understood [50], but it is fair to say that in the early (unresuscitated) stages there is generalized depression of cardiac function, manifesting clinically as a low cardiac output state. Following fluid therapy the situation often changes to a picture more typical of distributive shock - rapid bounding pulses, increased cardiac output and systemic vasodila- 
tion. Most ICUs no longer use pulmonary artery catheters to guide diagnosis and hemodynamic management in septic shock [51], and thus this is almost certainly the case for CRS- 5 too. Lastly, there is a growing appreciation of the value of echocardiography in the assessment and management of ICU patients with acute myocardial dysfunction [52]. Bedside assessment of myocardial filling status and systolic function is particularly helpful even if a full study is not obtainable.

Assessment of Renal Function. Diagnosis and assessment of renal function in sepsis-induced CRS- 5 is the same as in other causes of AKI. The clinical mainstay is still an acute change in serum creatinine levels, and consensus has been reached with the RIFLE [53], AKIN [54] and KDIGO criteria [55] essentially describing similar acute reductions in renal function. These systems also provide a means of assessing severity and have served to move the clinical research agenda forwards from recognition of the disease to risk assessment, assessment of treatment effect and transition to clinically important endpoints. In CRS-5, several novel biomarkers of AKI have been reported to have clinical utility. For reviews of these see Comnick and Ishani [56] and Cruz et al. [57]. Several new markers of renal function and AKI risk prediction are now in clinical use in Europe and time will tell if they have true clinical utility. Cystatin C is still the only new biomarker approved for diagnostic assessment in the USA at the time of writing. For the time being at least therefore it is likely that RIFLE, AKIN and KDIGO criteria using serum creatinine and urine output will continue to be used for diagnosis and monitoring of AKI in CRS-5.

\section{b. To Assess Progression and Resolution of Organ Dysfunction}

Risk Prediction. Once the diagnosis of septic shock and CRS-5 is made, organ function has been assessed and the patient is (hopefully) recovering, attention turns to risk prediction and protection of further deteriorations in organ function. In AKI, there are new data suggesting that biomarkers of cell cycle arrest may be able to predict which patients will progress to severe AKI within a few days [93]. These data will need to be confirmed in clinical practice, but the idea that a simple blood test may help clinicians identify those at increased risk of severe disease is certainly attractive. For cardiac risk prognostication, septic shock survivors have been shown to have lower ejection fractions and higher end-diastolic volumes when compared with patients who died, which appears to suggest a possible protective effect of myocardial depression [58]. Rudiger and Singer [50] provide a good discussion of the mechanisms underlying cardiac dysfunction in sepsis. 
Table 3. Therapeutic interventions in sepsis-induced CRS-5

Disease modification

Removal of inflammatory mediators

Rationale based on targeting removal of inflammatory molecules as molecular weight of most cytokines (e.g. TNF) ranges from 17,000-50,000

Cytokine removal is variable and depends on operational characteristics

Removal can be enhanced by

- Increasing permeability of membrane (high cut-off membranes, plasma filtration)

- Increasing convection (HVHF, pulse HVHF)

- Utilizing adsorption (PMMA CHDF, polymyxin, Osiris membrane)

Immunomodulation

Rationale based on targeting cellular elements of disease response

- Immunoparalysis

- Apoptosis

- Neutrophil activation

Strategies include

- Endotoxin removal by selective binding to polymyxin

- Anticoagulant-based strategies (citrate enabled selective cytophoretic device)

- Plasma filtration coupled to binding to sorbents

Organ support

\section{Cardiac}

Cardiac dysfunction contributes to shock and poor tissue perfusion

Increased vascular permeability results in maldistribution of fluid in various compartments Strategies include

- lonotropes

- Vasopressors

- Vasodilators

Renal

Renal dysfunction contributes to organ failure

- Solute clearance

- Fluid management

- Acid base and electrolyte homeostasis

Strategies include

- Diuretics

- RRT

\section{Q3. What Are the Targets and Best Strategies for Therapeutic Intervention?}

Therapeutic interventions in CRS-5 can be focused on disease modification, management of cardiac and renal dysfunction and their consequences (table 3 ). Decision for interventions should be based on knowledge of the phase of disease, the underlying pathophysiology and anticipated course. 


\section{a. Management of Underlying Disease}

General Principles. As shown in figure 3, patients in the hyperacute phase will have an emphasis on resuscitation and stabilization of hemodynamic factors coupled with source control. Several publications, e.g. the 'surviving sepsis' guidelines, provide recommendations for the initial management of patients with sepsis and septic shock [51]. Maintaining hemodynamic stability and tissue perfusion are key components for preventing CRS-5 in the hyperacute phase. However, source control and adequate antibiotic coverage need to be addressed. As discussed earlier, often fluid resuscitation results in fluid accumulation and continued maintenance fluids can contribute to fluid overload with deleterious consequences [59-62]. Thus avoiding iatrogenic complications from resuscitation and recognition of failing organ function are critical to reduce the development of CRS-5.

Disease Modification. The pathophysiology and molecular events of sepsis offers several therapeutic targets [63]. As shown in figure 1 the systemic inflammatory response with PAMPS and DAMPS sets into motion the release of several circulating cytokines [49]. Physical removal of cytokines and immunomodulation are the two main approaches that have been tried and are currently being assessed for disease modification based on extracorporeal techniques (table 3). Removal is based on the rationale that cytokine burden can be reduced by utilizing convection [64], high-volume hemofiltration [65, 66], high-permeability membranes [67, 68] or adsorption [69]. High-volume hemofiltration has not been very successful $[66,70]$, however limited studies have shown benefit with high-permeability membranes and adsorption [71]. An alternate approach is to target cellular elements of the disease response including immunoparalysis, apoptosis and neutrophil activation through endotoxin removal with a polymyxin filter [72] or a citrate anticoagulant-based selective cytopheretic device [73]. Both these techniques have been successful in small pilot studies and pivotal trials are underway. Plasma filtration coupled with sorbents has been studied in experimental models and clinical studies are being initiated $[74,75]$.

\section{c. Treatment of Cardiac and Renal Dysfunction}

Cardiac Support. Managing cardiac dysfunction in the hyperacute and acute phases requires a multipronged approach for maintaining adequate filling pressures with fluid resuscitation and the judicious uses of vasopressors, vasodilators and ionotropes. Several studies have evaluated the effect of vasopressors to restore blood pressure but can decrease cardiac output (increasing afterload), especially if with coexisting hypovolemia. Vasodilators increase cardiac output, especially in conditions with impaired contractility. Norepinephrine is preferred vasoconstrictor $(\alpha$-adrenergic effects with some inotropic effects via its moderate $\beta$-adrenergic 
effects). Phosphodiesterase inhibitors combined inotropic and vasodilating effects and (likely) less increase myocardial oxygen requirements than other inotropic agents. Vasopressin, strong vasoconstrictor, excessive increase in arterial pressure but has detrimental effects on cardiac output and splanchnic perfusion [76]. Recently, levosimendan has been shown to be of benefit in decompensated heart failure to improve cardiac performance and achieve a diuresis [77, 78]. Further studies are needed with this agent to determine if it can be used to ameliorate CRS-5.

Renal Support. Currently there are no specific drug-based interventions for renal dysfunction in sepsis [79]. General supportive measures include avoidance of nephrotoxic agents, maintenance of an adequate perfusion pressure and intervention with dialysis [80]. Several studies have evaluated the role of various vasopressor agents in improving renal function in sepsis $[81,82]$. There is no role for dopamine to improve renal hemodynamics/function $[81,83]$, and there have been limited studies with fenoldopam $[84,85]$. Norepinephrine increases systemic blood pressure but decreases renal perfusion in normal conditions; NE increases systemic blood pressure and renal perfusion in endotoxic conditions [86]. Vasopressin (natural antidiuretic effects) paradoxically increases urine output and (?) creatinine clearance in patients with septic shock [87-88]. It is currently unclear what the target systemic and intrarenal blood pressure should be to optimize renal function [89].

Management of Consequences: Involvement of the heart and kidney in septic shock portrays special challenges as increasing requirements for vasopressor and ionotrope support are coupled with oliguric states resulting in fluid accumulation and overload. Diuretics have limited roles [90] and RRT particularly with continuous renal replacement should be considered and implemented early [80]. There is some emerging evidence that implementing ultrafiltration early in the course of septic shock may improve outcomes, however further studies are needed to validate these concepts. Maintaining acid base balance and providing adequate 'space' for nutritional support are additional indications for early intervention with RRT.

\section{Research Needs}

More detailed epidemiological data...

- time course of developing CRS before/after ICU

- better definition/staging of epidemiological data, i.e. application of RIFLE/ AKIN criteria.

- Mechanistic/animal studies with...

- clinically relevant models 
- delineation between effects of systemic insult (sepsis) versus organ crosstalk

- application of definition/staging criteria to better compare animal models.

- More detailed observational studies to identify...

- pathways that are associated with development of CRS 5 (pro-/antiinflammatory pathways activation/role of RAAS)

- site of injury and its effects/prognostic role (glomerular vs. tubular, RV vs. LV failure).

- Experimental studies to elucidate cross-talk between heart and kidney during catastrophic conditions like sepsis, or less dramatic cases like chemo effecting heart and kidney (adriamycin, cisplatin).

- Elucidating these mechanisms could reveal novel biomarkers and improved therapy CRS-5.

- Diagnostic tests: There is a great need to assess the utility of different biomarkers of cardiac and renal dysfunction individually and in combination to diagnose and monitor the progress of CRS-5. These will likely be different for acute and chronic disorders, e.g. sepsis vs. cirrhosis. Techniques to measure biomarker panels and standardized reporting require further research.

\section{Conclusions}

CRS-5 is a complex condition which can result from several different conditions that vary in the time and severity. Consequently, it is challenging to attribute the condition to a single pathophysiological mechanism. The timing and sequence of dysfunction of the heart and kidney are influenced by the state of underlying health and the nature and duration of the precipitating condition.

\section{References}

1 Ronco C, McCullough PA, Anker SD, Anand I, Aspromonte N, Bagshaw SM, Bellomo R, Berl T, Bobek I, Cruz DN, Daliento L, Davenport A, Haapio M, Hillege H, House A, Katz NM, Maisel A, Mankad S, Zanco P, Mebazaa A, Palazzuoli A, Ronco F, Shaw A, Sheinfeld G, Soni S, Vescovo G, Zamperetti N, Ponikowski P, Acute Dialysis Quality Initiative (ADQI) Consensus Group: Cardiorenal syndromes: an executive summary from the consensus conference of the Acute Dialysis Quality Initiative (ADQI). Contrib Nephrol. Basel, Karger, 2010, vol 165, pp 54-67.
2 Lundy DJ, Trzeciak S: Microcirculatory dysfunction in sepsis. Crit Care Clin 2009;25: 721-731, viii.

3 Trzeciak S, et al: Early microcirculatory perfusion derangements in patients with severe sepsis and septic shock: relationship to hemodynamics, oxygen transport, and survival. Ann Emerg Med 2007;49:88-98, 98.e1-e2.

4 Sakr Y, et al: Persistent microcirculatory alterations are associated with organ failure and death in patients with septic shock. Crit Care Med 2004;32:1825-1831. 
5 Jardin F, et al: Sepsis-related cardiogenic shock. Crit Care Med 1990;18:1055-1060.

6 Parker MM, et al: Profound but reversible myocardial depression in patients with septic shock. Ann Intern Med 1984;100:483-490.

$>7$ Lambermont B, et al: Effects of endotoxic shock on right ventricular systolic function and mechanical efficiency. Cardiovasc Res 2003;59:412-418.

$>8$ Dhainaut JF, et al: Coronary hemodynamics and myocardial metabolism of lactate, free fatty acids, glucose, and ketones in patients with septic shock. Circulation 1987;75:533541.

$\checkmark 9$ Niederbichler AD, et al: An essential role for complement $\mathrm{C} 5 \mathrm{a}$ in the pathogenesis of septic cardiac dysfunction. J Exp Med 2006; 203:53-61.

10 Kumar A, et al: Tumor necrosis factor- $\alpha$ and interleukin- $1 \beta$ are responsible for in vitro myocardial cell depression induced by human septic shock serum. J Exp Med 1996; 183:949-958.

11 Torre-Amione G, et al: Proinflammatory cytokine levels in patients with depressed left ventricular ejection fraction: a report from the Studies of Left Ventricular Dysfunction (SOLVD). J Am Coll Cardiol 1996;27:12011206.

12 Benes J, et al: Searching for mechanisms that matter in early septic acute kidney injury: an experimental study. Crit Care 2011;15:R256.

13 Wan L, et al: Angiotensin II in experimental hyperdynamic sepsis. Crit Care 2009; 13:R190.

14 Bougle A, Duranteau J: Pathophysiology of sepsis-induced acute kidney injury: the role of global renal blood flow and renal vascular resistance. Contrib Nephrol. Basel, Karger, 2011, vol 174, pp 89-97.

15 Lerolle N, et al: Renal failure in septic shock: predictive value of Doppler-based renal arterial resistive index. Intensive Care Med 2006;32:1553-1559.

16 Murugan R, et al: Acute kidney injury in non-severe pneumonia is associated with an increased immune response and lower survival. Kidney Int 2010;77:527-535.

17 Papaioannou VE, et al: Relation of heart rate variability to serum levels of C-reactive protein, interleukin- 6 and -10 in patients with sepsis and septic shock. J Crit Care 2009; 24:625.e1-e7.
18 Schmidt H, et al: Autonomic dysfunction predicts both 1- and 2-month mortality in middle-aged patients with multiple organ dysfunction syndrome. Crit Care Med 2008; 36:967-970.

19 Tateishi Y, et al: Depressed heart rate variability is associated with high IL-6 blood level and decline in the blood pressure in septic patients. Shock 2007;28:549-553.

20 Ramchandra R, et al: Septic shock induces distinct changes in sympathetic nerve activity to the heart and kidney in conscious sheep. Am J Physiol Regul Integr Comp Physiol 2009;297:R1247-R1253.

21 Meijvis SC, et al: Prognostic value of serum angiotensin-converting enzyme activity for outcome of community-acquired pneumonia Clin Chem Lab Med 2011;49:1525-1532.

22 Nakada TA, et al: Association of angiotensin II type 1 receptor-associated protein gene polymorphism with increased mortality in septic shock. Crit Care Med 2011;39:16411648.

23 Doerschug KC, et al: Renin-angiotensin system activation correlates with microvascular dysfunction in a prospective cohort study of clinical sepsis. Crit Care 2010;14:R24.

24 Shen L, et al: Losartan prevents sepsisinduced acute lung injury and decreases activation of nuclear factor- $\kappa \mathrm{B}$ and mitogenactivated protein kinases. Shock 2009;31: 500-506

25 Hagiwara S, et al: Effects of an angiotensinconverting enzyme inhibitor on the inflammatory response in in vivo and in vitro models. Crit Care Med 2009;37:626-633.

26 Nitescu N, Grimberg E, Guron G: Low-dose candesartan improves renal blood flow and kidney oxygen tension in rats with endotoxin-induced acute kidney dysfunction. Shock 2008;30:166-172.

27 Mortensen EM, et al: Impact of previous statin and angiotensin II receptor blocker use on mortality in patients hospitalized with sepsis. Pharmacotherapy 2007;27:1619-1626.

28 Soni A, et al: Adrenal insufficiency occurring during septic shock: incidence, outcome, and relationship to peripheral cytokine levels. Am J Med 1995;98:266-271.

29 Annane D, et al: Corticosteroids in the treatment of severe sepsis and septic shock in adults: a systematic review. JAMA 2009;301: 2362-2375. 
30 Sligl WI, et al: Safety and efficacy of corticosteroids for the treatment of septic shock: a systematic review and meta-analysis. Clin Infect Dis 2009;49:93-101.

31 Kelly KJ: Distant effects of experimental renal ischemia/reperfusion injury. J Am Soc Nephrol 2003;14:1549-1558.

32 Grams ME, Rabb H: The distant organ effects of acute kidney injury. Kidney Int 2012;81: 942-948.

33 Burchill L, et al: Acute kidney injury in the rat causes cardiac remodelling and increases angiotensin-converting enzyme 2 expression. Exp Physiol 2008;93:622-630.

34 Tokuyama H, et al: Macrophage infiltration and cellular proliferation in the non-ischemic kidney and heart following prolonged unilateral renal ischemia. Nephron Physiol 2007; 106:54-62.

35 Hassoun HT, et al: Kidney ischemia-reperfusion injury induces caspase-dependent pulmonary apoptosis. Am J Physiol Renal Physiol 2009;297:F125-F137.

36 Grigoryev DN, et al: The local and systemic inflammatory transcriptome after acute kidney injury. J Am Soc Nephrol 2008;19:547558.

37 Feltes CM, et al: Pulmonary endothelial cell activation during experimental acute kidney injury. Shock 2011;36:170-176.

38 Liu M, et al: Acute kidney injury leads to inflammation and functional changes in the brain. J Am Soc Nephrol 2008;19:1360-1370.

39 Celes MR, Prado CM, Rossi MA: Sepsis: going to the heart of the matter. Pathobiology 2013;80:70-86.

40 Doi K, et al: Animal models of sepsis and sepsis-induced kidney injury. J Clin Invest 2009; 119:2868-2878.

41 Chopra M, et al: Modulation of myocardial mitochondrial mechanisms during severe polymicrobial sepsis in the rat. PLoS One 2011;6:e21285.

42 Liu M, et al: Transcription factor Nrf2 is protective during ischemic and nephrotoxic acute kidney injury in mice. Kidney Int 2009; 76:277-285.

43 Stengl M, et al: Reduced L-type calcium current in ventricular myocytes from pigs with hyperdynamic septic shock. Crit Care Med 2010;38:579-587.
44 Good DW, George T, Watts BA, 3rd: Tolllike receptor 2 mediates inhibition of $\mathrm{HCO}_{3}{ }^{-}$ absorption by bacterial lipoprotein in medullary thick ascending limb. Am J Physiol Renal Physiol 2010;299:F536-F544.

45 Hsiao HW, et al: The decline of autophagy contributes to proximal tubular dysfunction during sepsis. Shock 2012;37:289-296.

46 Schreiber A, et al: Acute endotoxemia in mice induces downregulation of megalin and cubilin in the kidney. Kidney Int 2012;82:53-59.

47 Bouchard J, et al: Fluid accumulation, survival and recovery of kidney function in critically ill patients with acute kidney injury. Kidney Int 2009;76:422-427.

48 American College of Chest Physicians/Society of Critical Care Medicine Consensus Conference: Definitions for sepsis and organ failure and guidelines for the use of innovative therapies in sepsis. Crit Care Med 1992;20:864874.

49 Reinhart K, et al: New approaches to sepsis: molecular diagnostics and biomarkers. Clin Microbiol Rev 2012;25:609-634.

50 Rudiger A, Singer M: Mechanisms of sepsisinduced cardiac dysfunction. Crit Care Med 2007;35:1599-1608.

51 Dellinger RP, et al: Surviving Sepsis Campaign: international guidelines for management of severe sepsis and septic shock: 2008 . Crit Care Med 2008;36:296-327.

52 Vignon P, et al: Hand-held echocardiography with Doppler capability for the assessment of critically-ill patients: is it reliable? Intensive Care Med 2004;30:718-723.

53 Bellomo R, et al: Acute renal failure - definition, outcome measures, animal models, fluid therapy and information technology needs: the Second International Consensus Conference of the Acute Dialysis Quality Initiative (ADQI) Group. Crit Care 2004;8:R204-R212.

54 Mehta RL, et al: Acute Kidney Injury Network: report of an initiative to improve outcomes in acute kidney injury. Crit Care 2007; 11:R31.

-55 KDIGO Clinical Practice Guideline for Acute Kidney Injury: Summary of recommendation statements. Kidney Int 2012;suppl 2:8-12.

- 56 Comnick M, Ishani A: Renal biomarkers of kidney injury in cardiorenal syndrome. Curr Heart Fail Rep 2011;8:99-105.

57 Cruz DN, et al: Role of biomarkers in the diagnosis and management of cardio-renal syndromes. Semin Nephrol 2012;32:79-92. 
58 Calvin JE, Driedger AA, Sibbald WJ: An assessment of myocardial function in human sepsis utilizing ECG gated cardiac scintigraphy. Chest 1981;80:579-586.

59 Liu KD, et al: Acute kidney injury in patients with acute lung injury: impact of fluid accumulation on classification of acute kidney injury and associated outcomes. Crit Care Med 2011;39:2665-2671.

60 Macedo E, et al: Fluid accumulation, recognition and staging of acute kidney injury in critically-ill patients. Crit Care 2010;14:R82.

61 Bellomo R, et al: Fluid management in septic acute kidney injury and cardiorenal syndromes. Contrib Nephrol. Basel, Karger, 2010, vol 165, pp 206-218.

62 Boyd JH, et al: Fluid resuscitation in septic shock: a positive fluid balance and elevated central venous pressure are associated with increased mortality. Crit Care Med 2011;39: 259-265.

63 Zarjou A, Agarwal A: Sepsis and acute kidney injury. J Am Soc Nephrol 2011;22:999-1006.

64 De Vriese AS, et al: Cytokine removal during continuous hemofiltration in septic patients. J Am Soc Nephrol 1999;10:846-853.

65 Tapia P, et al: Effectiveness of short-term 6-hour high-volume hemofiltration during refractory severe septic shock. J Trauma Acute Care Surg 2012;72:1228-1238.

66 Honore PM, et al: High-volume hemofiltration in sepsis and SIRS: current concepts and future prospects. Blood Purif 2009;28:1-11.

67 Matson J, Zydney A, Honore PM: Blood filtration: new opportunities and the implications of systems biology. Crit Care Resusc 2004;6:209-217.

68 Uchino S, et al: Cytokine dialysis: an ex vivo study. ASAIO J 2002;48:650-653.

69 Nakada TA, et al: Continuous hemodiafiltration with PMMA hemofilter in the treatment of patients with septic shock. Mol Med 2008;14:257-263.

$>70$ Peng Z, et al: Evaluation of the effects of pulse high-volume hemofiltration in patients with severe sepsis: a preliminary study. Int J Artif Organs 2010;33:505-511.

71 Nakamura M, et al: Treatment of severe sepsis and septic shock by CHDF using a PMMA membrane hemofilter as a cytokine modulator. Contrib Nephrol. Basel, Karger, 2010, vol 166, pp 73-82.
72 Cruz DN, et al: Early use of polymyxin B hemoperfusion in abdominal septic shock: the EUPHAS randomized controlled trial. JAMA 2009;301:2445-2452.

73 Humes HD, et al: A selective cytopheretic inhibitory device to treat the immunological dysregulation of acute and chronic renal failure. Blood Purif 2010;29:183-190.

74 Formica M, et al: Coupled plasma filtration adsorption. Contrib Nephrol. Basel, Karger, 2007, vol 156, pp 405-410.

75 Rimmele T, Kellum JA: Clinical review: blood purification for sepsis. Crit Care 2011;15:205.

76 Faivre V, et al: Cardiac and renal effects of levosimendan, arginine vasopressin, and norepinephrine in lipopolysaccharide-treated rabbits. Anesthesiology 2005; 103:514-521.

77 Hou ZQ, et al: Effect of levosimendan on estimated glomerular filtration rate in hospitalized patients with decompensated heart failure and renal dysfunction. Cardiovasc Ther 2012 (E-pub ahead of print).

78 Ristikankare A, et al: Effects of levosimendan on renal function in patients undergoing coronary artery surgery. J Cardiothorac Vasc Anesth 2012;26:591-595.

79 May CN, et al: Novel targets for sepsisinduced kidney injury: the glomerular arterioles and the sympathetic nervous system. Exp Physiol 2012;97:1168-1177.

80 Chou YH, et al: Impact of timing of renal replacement therapy initiation on outcome of septic acute kidney injury. Crit Care 2011; 15:R134.

-81 Bellomo R, Wan L, May C: Vasoactive drugs and acute kidney injury. Crit Care Med 2008; 36(suppl):S179-S186.

82 Brienza N, et al: A comparison between fenoldopam and low-dose dopamine in early renal dysfunction of critically ill patients. Crit Care Med 2006;34:707-714.

83 Schmoelz M, et al: Comparison of systemic and renal effects of dopexamine and dopamine in norepinephrine-treated septic shock. J Cardiothorac Vasc Anesth 2006;20: 173-178.

84 Cobas M, et al: Fenoldopam in critically ill patients with early renal dysfunction. A crossover study. Cardiovasc Ther 2011;29: 280-284.

85 Landoni G, et al: Fenoldopam in cardiac surgery-associated acute kidney injury. Int J Artif Organs 2008;31:561. 
86 Redfors B, et al: Effects of norepinephrine on renal perfusion, filtration and oxygenation in vasodilatory shock and acute kidney injury. Intensive Care Med 2011;37:60-67.

87 Guzman JA, Rosado AE, Kruse JA: Vasopressin vs. norepinephrine in endotoxic shock: systemic, renal, and splanchnic hemodynamic and oxygen transport effects. J Appl Physiol 2003;95:803-809.

88 Gordon AC, et al: The effects of vasopressin on acute kidney injury in septic shock. Intensive Care Med 2010;36:83-91.

89 Deruddre S, et al: Renal arterial resistance in septic shock: effects of increasing mean arterial pressure with norepinephrine on the renal resistive index assessed with Doppler ultrasonography. Intensive Care Med 2007; 33:1557-1562.
90 Nigwekar SU, Waikar SS: Diuretics in acute kidney injury. Semin Nephrol 2011;31:523534.

-91 Muciño-Bermejo J, Carrillo-Esper R, Uribe M, Méndez-Sánchez N: Acute kidney injury in critically ill cirrhotic patients: a review. Ann Hepatol 2012;11:301-310.

92 Wong F, et al: Working Party proposal for a revised classification system of renal dysfunction in patients with cirrhosis. Gut 2011;60: 702-709.

93 Kashani K, et al: Discovery and validation of cell cycle arrest biomarkers in human acute kidney injury. Crit Care 2013;17:R25. 